\title{
Somatic symptoms in adolescence as a predictor of severe mental illness in adulthood: a long-term community-based follow-up study
}

Hannes Bohman ${ }^{1,2,3^{*}}$, Sara B. Låftman ${ }^{4}$, Neil Cleland ${ }^{5}$, Mathias Lundberg ${ }^{3}$, Aivar Päären ${ }^{1}$ and Ulf Jonsson ${ }^{1,6}$

\begin{abstract}
Background: Somatic symptoms are common and costly for society and correlate with suffering and low functioning. Nevertheless, little is known about the long-term implications of somatic symptoms. The objective of this study was to assess if somatic symptoms in adolescents with depression and in their matched controls predict severe mental illness in adulthood by investigating the use of hospital-based care consequent to different mental disorders.

Methods: The entire school population of 16-17-year-olds in the city of Uppsala, Sweden, was screened for depression in 1991-1993 $(n=2300)$. Adolescents with positive screenings $(n=307)$ and matched non-depressed controls $(n=302)$ participated in a semi-structured diagnostic interview for mental disorders. In addition, 21 different self-rated somatic symptoms were assessed. The adolescents with depression and the matched non-depressed controls were engaged in follow-up through the National Patient Register 17-19 years after the baseline study $(n=375)$. The outcome measures covered hospital-based mental health care for different mental disorders according to ICD-10 criteria between the participants' ages of 18 and 35 years.

Results: Somatic symptoms were associated with an increased risk of later hospital-based mental health care in general in a dose-response relationship when adjusting for sex, adolescent depression, and adolescent anxiety (1 symptom: $\mathrm{OR}=1.63, \mathrm{Cl} 0.55-4.85 ; 2-4$ symptoms: $\mathrm{OR}=2.77,95 \% \mathrm{Cl} 1.04-7.39 ; \geq 5$ symptoms: $\mathrm{OR}=5.75,95 \% \mathrm{Cl}$ 1.98-16.72). With regards to specific diagnoses, somatic symptoms predicted hospital-based care for mood disorders when adjusting for sex, adolescent depression, and adolescent anxiety $(p<0.05)$. In adolescents with depression, somatic symptoms predicted later hospital-based mental health care in a dose-response relationship ( $p<0.01)$. In adolescents without depression, reporting at least one somatic symptom predicted later hospital-based mental health care $(p<0.05)$.

Conclusions: Somatic symptoms in adolescence predicted severe adult mental illness as measured by hospitalbased care also when controlled for important confounders. The results suggest that adolescents with somatic symptoms need early treatment and extended follow-up to treat these specific symptoms, regardless of co-occurring depression and anxiety.
\end{abstract}

\footnotetext{
*Correspondence: hannes.bohman@neuro.uu.se

1 Department of Neuroscience, Child and Adolescent Psychiatry, Uppsala

University, Box 593, 75124 Uppsala, Sweden

Full list of author information is available at the end of the article
} 


\section{Background}

The experience of somatic symptoms, such as gastrointestinal pain, headache, back pain and tiredness, is common in the general population $[1,2]$. Somatic symptoms are expensive in terms of direct costs for health care but also in a wider societal perspective due to decreased productivity [3, 4]. Research over the two past decades has documented that somatic symptoms are also common in community-based samples of children and adolescents, particularly among girls [5-7]. Children and adolescents suffering from somatic symptoms perform worse in school [8], are more often absent from school, and more often tend to have problematic social relations [9-11]. Somatic symptoms in children and adolescents are also associated with mental disorders such as anxiety and depression $[9,12-20]$ and with other severe concurrent psychiatric problems in a dose-response relationship-for example, conduct disorder, suicidal behavior, and experiences of multiple interpersonal conflicts $[15$, 21-23].

However, less is known about the long-term implications of somatic symptoms in childhood and adolescence and follow-up periods rarely stretch longer than until young adulthood. In particular, there is a lack of knowledge about the long-term outcomes of somatic symptoms when adjusted for concurrent mental disorders and other confounders [24]. Only a few studies have investigated the long-term interrelationship between somatic symptoms, depression and anxiety at both baseline and follow up [25, 26]. In addition, most of the previous longterm follow-up studies of somatic symptoms and later mental health outcomes have used self-reported measures of mental disorders at follow-up [24]. Thus, little is known about the potential severe implications of somatic symptoms in terms of, for example, the use of hospitalbased mental health care.

In a previous study, we followed up on adolescents with depression and somatic symptoms until they reached an adult age. We showed that adolescents with somatic symptoms had increased risks of adult depression, anxiety and other mental disorders, independent of concurrent adolescent depression and other confounders [27]. Despite having important findings, the previous study suffered from some limitations. The study relied on self-reported interview diagnoses rather than on clinical diagnoses. Depression was recorded retrospectively, thus introducing the possibility of recall bias. Depression and somatic symptoms were assessed both at baseline and at follow up, but anxiety was not included in the baseline analyses in this study. In addition, in the previous study, we did not investigate the severity of the mental disorders, e.g., the use of advanced health care. In the present study, we use register data that included diagnoses of hospital-based mental health care during the 17- to 19-year follow-up period. These data enabled us to investigate severe mental illness in terms of the use of advanced health care for mental disorders without the possibility of recall bias. The data also allowed us to assess the predictive power of somatic symptoms in adolescence, while adjusting for depression and anxiety in adolescence as well as sex and other potential confounders.

The aim of the current study was to test the hypothesis that adolescent somatic symptoms predict severe mental illness in adulthood. We address three research questions:

1. Are somatic symptoms in adolescents a predictor for later severe mental illness, measured by the use of adult hospital-based care for mental disorders, while also adjusting for adolescent depression and anxiety and other important confounders?

2. Are the number of concurrent somatic symptoms in depressed adolescents a predictor for later severe mental illness, measured by the use of adult hospitalbased care for mental disorders?

3. Are somatic symptoms in non-depressed adolescents a predictor for later severe mental illness, measured by the use of adult hospital-based care for mental disorders?

\section{Methods}

\section{Study population and procedure}

In 1991-1993, all first-year students in upper secondary school (16-17 years old) in the Swedish university town of Uppsala, with approximately 180,000 inhabitants, were asked to participate in a screening for depression [28]. School dropouts were also invited. Out of a total of 2465 individuals, 93\% $(n=2300)$ participated in the screening, which included two self-evaluations of depression: the Beck Depression Inventory-Child and the Centre for Epidemiological Studies-Depression Scale for Children [29]. Students with high scores and those who reported a suicide attempt were interviewed with the Diagnostic Interview for Children and Adolescents with a revised form according to the DSM-III-R (DICA-R-A) [30]. In all, 355 students in the screening were classified as suffering from depression and were accordingly selected for a diagnostic interview. For each depressed student, a same-sex classmate and with low scores in the screening was recruited into a comparison group. In total, 609 individuals $(n=307$ in the depressed group and $n=302$ in the control group) participated in the diagnostic interview and consented to be contacted for a future follow-up study. At the time of the interview, they also completed a range of self-rating measures, including the Somatic Symptom 
Checklist Instrument (SCI) on somatic symptoms. Some of the participants in the comparison group $(n=65)$ were retrospectively diagnosed with major depression or dysthymia occurring before the baseline study and consequently were included in the depression group. Some of the participants with positive screenings did not meet the criteria for a depressive disorder upon being interviewed for current and lifetime major depression or dysthymia and were in the present analyses relocated to the control group $(n=55)$. Approximately 15 years after the baseline study, the participants who had consented to a follow-up study were contacted and invited to a follow-up interview. They were also asked if they wanted to participate in studies that included health registers. Data were subsequently collected from health registers $17-19$ years after the baseline study. Among the 609 individuals who had participated in the diagnostic interview and who also had completed the SCI at baseline, approximately $70 \%$ participated in the follow-up interview. Of these, 375 individuals gave their written consent to be followed through the health registers $(n=182$ in the depression group and $\mathrm{n}=193$ in the control group). The procedure is outlined in Fig. 1. Further information about the follow-up study is provided elsewhere [27, 31].

\section{Adolescent depression}

Adolescent depression was defined as major depressive disorder (MDD) or dysthymia according to DICA-R-A [30] (see Fig. 1).

\section{Adolescent anxiety}

Adolescent anxiety was defined as any anxiety disorder according to DICA-R-A [30].

\section{Adolescent somatic symptoms}

The SCI is a Swedish version of the Psychosomatic Symptom Checklist [32]. The SCI assesses 22 items reflecting various somatic symptoms: tiredness, headache, feeling chilly, insomnia, eye tiredness, abdominal pain, dizziness, nausea, perspiration, appetite problem, breathing problem, polyuria, limb pain, itching, dry mouth, palpitation, constipation, fainting, regurgitation, chewing

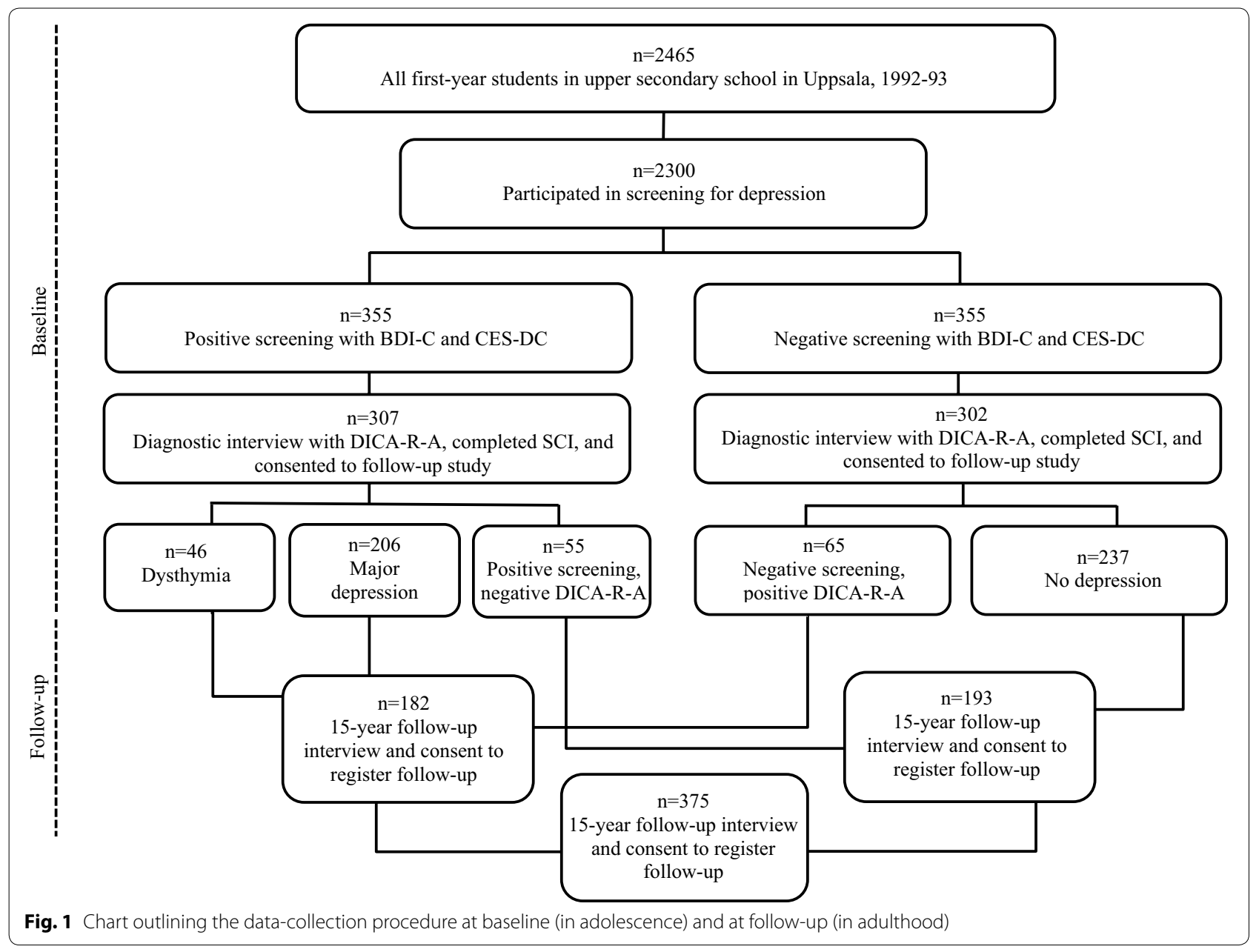


pain, and swallowing problems. Allergy was part of the checklist but was excluded from the analyses because it was considered a somatic disease rather than a symptom. The symptoms were graded in frequency $(0=$ never, $1=$ monthly, $2=$ weekly, $3=$ several times a week, and $4=$ daily $)$, and intensity $(0=$ no problem, $1=$ minor, $2=$ moderate,$\quad 3=$ troublesome, and $4=$ extremely troublesome), for the last month. The questionnaire has been used in previous publications [12, 23, 27]. A somatic symptom was recorded when the frequency and intensity were multiplied to yield a score $\geq 6$ (e.g., $2 \times 3$ : weekly $\times$ troublesome symptoms). Such a scoring approach excluded minor problems and the possibility that monthly premenstrual symptoms would be recorded as positive. The same cut-off has been used in earlier publications [23, 27].

In the analyses of the control group, somatic symptoms were categorized as 0 vs. $\geq 1$ symptoms (a more fine-grained categorization was not possible due to small numbers in the cells). In the analyses of individuals with adolescent depression, four categories of somatic symptoms were created: $0,1,2-4$, and $\geq 5$ symptoms - a categorization that was grounded in our previous analyses of the same data material, where $\geq 5$ somatic symptoms were found to characterize a threshold value in the prediction of mental health outcomes in adulthood [27].

\section{Confounders}

A set of potential confounders, which may potentially have affected both somatic symptoms at baseline and mental disorders in adulthood, were used to adjust the analyses. Information on conflicts between parents, conflicts with parents, economic hardship, parental unemployment, and somatic illness collected at baseline through the Children's Life Inventory [33] was included. In addition, we included information on physical/sexual abuse in childhood collected retrospectively in the follow-up study [31]. Conflicts between parents and conflicts with parents were shown to be significantly related to major depression at baseline [34] as well as to somatic symptoms at baseline [23], and analyses of the follow-up data demonstrated that conflicts with parents and physical/sexual abuse in childhood were associated with mental disorders in adulthood [31]. Socioeconomic status in the family of origin had been shown to be associated with mental disorders in adulthood [35]; therefore, measures of economic hardship and parental unemployment collected at baseline were included. In order to account for the fact that some somatic symptoms might have had a medical explanation (i.e. due to somatic illness), a measure of somatic illness reported in adolescence was included. The variable was created from two items from the Children's Life Inventory [33]: "I have been severely ill or injured", and "I have been hospitalized more than one week", with the possible response categories "During the past year" and "Earlier in life". The measure of somatic illness was defined by a positive record on at least one of these two items, i.e., self-reported somatic illness or injury some time in life until baseline and/ or the adolescent's report on having ever been hospitalized more than 1 week some time in life until baseline. The data did however not include any information about specific somatic diagnoses.

\section{Outcomes}

The Swedish National Health and Welfare Board maintains the official registers concerning health and sickness in Sweden. The national patient register was used in the present study from 1992 until 2009. The national patient register includes data on inpatient care and outpatient hospital-based care. With regard to inpatient care, the register data cover almost all inpatient visits since 1987. With regard to hospital-based outpatient care, outpatient visits have been registered since 2001, but only a part of the data is covered during the follow-up period. Hospital-based mental health care diagnoses were classified according to ICD-10 criteria-specifically, the codes F10-F69 were used to define hospital-based mental health care. For more detailed analyses, the diagnoses were also divided into different general categories: F10F19, mental and behavioral disorders due to psychoactive substance use; F20-F29, schizophrenia, schizotypal and delusional disorders; F30-F39, mood disorders; F40F48, neurotic, stress-related and somatoform disorders (including all anxiety disorders); F50-F59, behavioral syndromes associated with physiological disturbances and physical factors; and F60-F69, disorders of adult personality and behavior.

\section{Data analysis}

Binary logistic regression analyses were performed to assess the association of somatic symptoms in adolescence with later hospital-based mental health care. Adjustments were made for adolescent depression and anxiety, sex and other potential confounders. Odds ratios with 95\% confidence intervals were reported. In the descriptive analyses of somatic symptoms and specific mental health care diagnoses, when several categories of somatic symptoms were compared, linear-by-linear associations were used to calculate linear relationships. To compare the groups of individuals with 0 and $\geq 1$ somatic symptoms at baseline, respectively, the Fisher's exact test was used. Stata version 15 (StataCorp, College Station, TX) was used. 


\section{Results}

Descriptive statistics for the pooled sample and, separately, for individuals without and with adolescent depression are presented in Table 1. Adolescents with depression had more concurrent somatic symptoms on average compared to the controls (3.10 vs. 1.27, $\mathrm{p}<0.001$ ). (Details on the prevalence of specific somatic symptoms are provided in Additional file 1: Appendix S1). All of the included potential confounders were substantially more common among individuals with adolescent depression than among controls without adolescent depression. In adulthood, any hospital-based mental health care diagnosis was significantly more common in the depressed group than in the control group $(\mathrm{OR}=2.80, \mathrm{p}<0.01)$. This pattern was reflected in all specific diagnoses, although the difference between groups was statistically significant only for mood disorders. As seen in Table 1, however, when distinguishing any hospital-based mental health care at the level of the specific diagnosis, the absolute numbers of cases were small.

In a series of binary logistic regression analyses in the pooled sample of individuals with and without adolescent

Table 1 Descriptive statistics for the pooled sample and separately for adolescents without depression (control group) and adolescents with depression at baseline, and differences between these groups (reference category $=$ control group)

\begin{tabular}{|c|c|c|c|c|c|}
\hline & All $(n=375)$ & $\begin{array}{l}\text { Adolescents } \\
\text { without depression } \\
(n=182)\end{array}$ & $\begin{array}{l}\text { Adolescents } \\
\text { with depression } \\
(n=193)\end{array}$ & OR & $95 \% \mathrm{Cl}$ \\
\hline & $\%(n)$ & $\%(n)$ & $\%(n)$ & & \\
\hline \multicolumn{6}{|l|}{ Sex } \\
\hline Males (ref.) & $19.7(74)$ & $22.0(40)$ & $17.6(34)$ & 1.00 & - \\
\hline \multirow[t]{2}{*}{ Females } & $80.3(301)$ & $78.0(142)$ & $82.4(159)$ & 1.32 & $0.79-2.19$ \\
\hline & Mean (s.d.) & Mean (s.d.) & Mean (s.d.) & $t$ test & \\
\hline \multicolumn{6}{|l|}{ Adolescence } \\
\hline \multirow[t]{2}{*}{ Number of concurrent somatic symptoms } & $2.21(2.36)$ & $1.27(1.76)$ & $3.10(2.50)$ & $p<0.001$ & \\
\hline & Mean (s.d.) & Mean (s.d.) & Mean (s.d.) & $x^{2}$ & $\mathbf{p}$ \\
\hline 0 & $27.5(103)$ & $41.7(76)$ & $14.0(27)$ & & \\
\hline 1 & $23.2(87)$ & $26.4(48)$ & $20.2(39)$ & & \\
\hline $2-4$ & $34.4(129)$ & $27.5(50)$ & $40.9(79)$ & & \\
\hline \multirow[t]{2}{*}{$\geq 5$} & $14.9(56)$ & $4.4(8)$ & $24.9(48)$ & 59.06 & $<0.001$ \\
\hline & $\%(n)$ & $\%(n)$ & $\%(n)$ & OR & $95 \% \mathrm{Cl}$ \\
\hline Adolescent anxiety & $28.0(105)$ & $7.1(13)$ & $47.7(92)$ & $11.84^{* * *}$ & $6.30-22.25$ \\
\hline Conflicts between parents & $20.8(78)$ & $11.5(21)$ & $29.5(57)$ & $3.21^{* * *}$ & $1.85-5.57$ \\
\hline Conflicts with parents & $19.5(73)$ & $7.7(14)$ & $30.6(59)$ & $5.28^{* * *}$ & $2.83-9.87$ \\
\hline Physical abuse & $12.3(46)$ & $6.0(11)$ & $18.1(35)$ & $3.44^{* *}$ & $1.69-7.01$ \\
\hline Economic hardship & $6.9(26)$ & $1.7(3)$ & $11.9(23)$ & $8.07^{* *}$ & $2.38-27.38$ \\
\hline Parental unemployment & $11.5(43)$ & $6.6(12)$ & $16.1(31)$ & $2.71^{* *}$ & $1.35-5.46$ \\
\hline \multirow[t]{2}{*}{ Somatic illness } & $20.3(76)$ & $13.7(25)$ & $26.4(51)$ & $2.26^{* *}$ & $1.33-3.83$ \\
\hline & $\%(n)$ & $\%(n)$ & $\%(n)$ & OR & $95 \% \mathrm{Cl}$ \\
\hline \multicolumn{6}{|l|}{ Adulthood } \\
\hline Any hospital-based mental health care diagnosis & $15.2(57)$ & $8.8(16)$ & $21.2(41)$ & $2.80^{* *}$ & $1.51-5.19$ \\
\hline $\begin{array}{l}\text { F10-F19 Mental and behavioral disorders due to psychoactive } \\
\text { substance use }\end{array}$ & $2.1(8)$ & $1.7(3)$ & $2.6(5)$ & 1.59 & $0.37-6.74$ \\
\hline F20-F29 Schizophrenia, schizotypal and delusional disorders & $0.5(2)$ & $0.0(0)$ & $1.0(2)$ & - & - \\
\hline F30-F39 Mood disorders & $7.2(27)$ & $4.4(8)$ & $9.8(19)$ & $2.38^{*}$ & $1.01-5.57$ \\
\hline F40-F48 Neurotic, stress-related and somatoform disorders & $9.1(34)$ & $6.6(12)$ & $11.4(22)$ & 1.82 & $0.87-3.80$ \\
\hline $\begin{array}{l}\text { F50-F59 Behavioral syndromes associated with physiological } \\
\text { disturbances and physical factors }\end{array}$ & $1.9(7)$ & $1.7(3)$ & $2.1(4)$ & 1.26 & $0.28-5.72$ \\
\hline F60-F69 Disorders of adult personality and behavior & $1.6(6)$ & $1.1(2)$ & $2.1(4)$ & 1.90 & $0.34-10.53$ \\
\hline
\end{tabular}

*** $p<0.001,{ }^{* *} p<0.01,{ }^{*} p<0.05$ 
depression, the association between somatic symptoms in adolescence and adult hospital-based mental health care was analyzed (Table 2). The crude model included only the categories of somatic symptoms, showing that the number of somatic symptoms was associated with any hospital-based mental health care in a step-wise manner (for 2-4 symptoms OR $=3.51,95 \%$ CI $1.37-8.98$, and for $\geq 5$ somatic symptoms OR $=8.30,95 \%$ CI 3.08-22.41). Model 1 added adolescent depression, adolescent anxiety, and sex. The estimates for the categories of somatic symptoms were attenuated, but those corresponding to $2-4$ and $\geq 5$ somatic symptoms remained robust and statistically significant $(\mathrm{OR}=2.77,95 \%$ CI 1.04-7.39, and $\mathrm{OR}=5.75$, 95\% CI 1.98-16.72, respectively). Model 2 added a number of potential confounders measured in adolescence, i.e., conflicts between parents, conflicts with parents, physical abuse, economic hardship, and parental unemployment. The association between $\geq 5$ somatic symptoms and any hospital-based mental health care diagnosis in adulthood remained robust and statistically significant $(\mathrm{OR}=5.03,95 \% \mathrm{CI} 1.66-15.28)$. To test whether the association between somatic symptoms in adolescence and hospital-based mental health care diagnosis in adulthood differed between adolescents with and without depression, an interaction term between somatic symptoms and adolescent depression was included. This was however not shown to be statistically significant $(p=0.587)$. Furthermore, to assess whether certain somatic symptoms were especially powerful predictors of later hospital-based mental health care, we also performed analyses of the associations between each specific somatic symptom and hospital-based mental health care in adulthood. Those that turned out to be statistically significant were tiredness, insomnia, headache, limb pain, abdominal pain, nausea and perspiration without exercise (see Additional file 1: Appendix S2).
Next, we present analyses of the associations between somatic symptoms and specific psychiatric diagnoses. As reported in Table 1, multiple somatic symptoms were more common among adolescents with depression than among those without depression. Therefore, for adolescents with depression we performed analyses of the number of somatic symptoms and psychiatric diagnoses (presented in Table 3), whereas for adolescents without depression we assessed the association between the presence of any $(\geq 1)$ somatic symptom and psychiatric diagnoses (presented in Table 4).

Among individuals with adolescent depression, the likelihood of having received any hospital-based mental health care was associated with somatic symptoms in a linear manner $(\mathrm{p}<0.01)$ (Table 3$)$. Among the specific diagnoses, a statistically significant linear relationship with the number of somatic symptoms was only found for mood disorders $(p<0.01)$. Yet, for nearly all specific diagnoses (except for behavioral syndromes), hospitalbased mental health care was most prevalent in the category with five or more somatic symptoms.

The presence of adult hospital-based mental health care among individuals without adolescent depression (i.e., the controls), differentiated by the presence of somatic symptoms in adolescence, is presented in Table 3. Compared with the controls without somatic symptoms, those with $\geq 1$ somatic symptoms were more likely to have received hospital-based mental health care in adulthood $(2.6 \%$ vs. $13.2 \%$, respectively; $\mathrm{p}<0.05)$. Among the specific diagnoses, hospital-based care for neurotic, stress-related and somatoform disorders $(1.3 \%$ vs. $10.4 \% ; \mathrm{p}<0.05)$ differed significantly between the controls without and with one or more somatic symptoms in adolescence.

Next, we wanted to compare the strength of association of somatic symptoms, depression, and anxiety,

Table 2 Odds ratios and $95 \%$ confidence intervals from binary logistic regression analyses of any hospital-based mental health care diagnosis in the pooled sample, $n=375$

\begin{tabular}{|c|c|c|c|c|c|c|c|}
\hline & \multirow[t]{2}{*}{$\mathbf{n}$} & \multicolumn{2}{|l|}{ Crude $^{a}$} & \multicolumn{2}{|c|}{ Model $1^{b}$} & \multicolumn{2}{|c|}{ Model $2^{c}$} \\
\hline & & OR & $95 \% \mathrm{Cl}$ & OR & $95 \% \mathrm{Cl}$ & OR & $95 \% \mathrm{Cl}$ \\
\hline \multicolumn{8}{|c|}{ Number of somatic symptoms } \\
\hline 0 (ref.) & 103 & 1.00 & - & 1.00 & - & 1.00 & - \\
\hline 1 & 87 & 1.87 & $0.64-5.47$ & 1.63 & $0.55-4.85$ & 1.54 & $0.51-4.66$ \\
\hline $2-4$ & 129 & $3.51^{* *}$ & $1.37-8.98$ & $2.77^{*}$ & $1.04-7.39$ & 2.67 & $0.98-7.25$ \\
\hline$\geq 5$ & 56 & $8.30^{* * *}$ & $3.08-22.41$ & $5.75^{* *}$ & $1.98-16.72$ & $5.03^{* *}$ & $1.66-15.28$ \\
\hline
\end{tabular}

\footnotetext{
${ }^{a}$ Crude includes categories pertaining to the number of somatic symptoms

${ }^{b}$ Model 1 adds adolescent depression, adolescent anxiety, and sex

c Model 2 adds conflicts between parents, conflicts with parents, physical abuse, economic hardship, parental unemployment, and somatic illness

${ }^{* * *} p<0.001,{ }^{* *} p<0.01,{ }^{*} p<0.05$
} 
Table 3 Adult hospital-based mental health care diagnoses at follow-up among individuals with adolescent depression, respectively, and numbers of somatic symptoms

\begin{tabular}{|c|c|c|c|c|c|}
\hline \multirow[t]{3}{*}{ Number of somatic symptoms } & \multicolumn{5}{|c|}{$\begin{array}{l}\text { Individuals with adolescent depression } \\
(n=193)\end{array}$} \\
\hline & $\begin{array}{l}0 \\
(n=27)\end{array}$ & $\begin{array}{l}1 \\
(n=39)\end{array}$ & $\begin{array}{l}2-4 \\
(n=79)\end{array}$ & $\begin{array}{l}\geq 5 \\
(n=48)\end{array}$ & Linear by linear \\
\hline & $\%(n)$ & $\%(n)$ & $\%(n)$ & $\%(n)$ & \\
\hline Any hospital-based mental health care diagnosis & $14.8(4)$ & $10.3(4)$ & $19.0(15)$ & $37.5(18)$ & $p<0.01$ \\
\hline F10-F19 Mental and behavioral disorders due to psychoactive substance use & $3.7(1)$ & $0.0(0)$ & $1.3(1)$ & $6.3(3)$ & n.s. \\
\hline F20-F29 Schizophrenia, schizotypal and delusional disorders & $0.0(0)$ & $0.0(0)$ & $1.3(1)$ & $2.1(1)$ & n.s. \\
\hline F30-F39 Mood disorders & $0.0(0)$ & $2.6(1)$ & $11.4(9)$ & $18.8(9)$ & $p<0.01$ \\
\hline F40-F48 Neurotic, stress-related and somatoform disorders & $11.1(3)$ & $7.7(3)$ & $7.6(6)$ & $20.8(10)$ & n.s. \\
\hline $\begin{array}{l}\text { F50-F59 Behavioral syndromes associated with physiological disturbances and } \\
\text { physical factors }\end{array}$ & $0.0(0)$ & $2.6(1)$ & $3.8(3)$ & $0.0(0)$ & n.s. \\
\hline F60-F69 Disorders of adult personality and behavior & $0.0(0)$ & $0.0(0)$ & $2.5(2)$ & $4.2(2)$ & n.s. \\
\hline
\end{tabular}

Table 4 Adult hospital-based mental health care diagnoses at follow-up among individuals without adolescent depression, and numbers of somatic symptoms

\begin{tabular}{|c|c|c|c|}
\hline \multirow[t]{3}{*}{ Number of somatic symptoms } & \multicolumn{3}{|c|}{$\begin{array}{l}\text { Individuals without adolescent depression } \\
(n=182)\end{array}$} \\
\hline & $\begin{array}{l}0 \\
(n=76)\end{array}$ & $\begin{array}{l}\geq 1 \\
(n=106)\end{array}$ & Fisher's exact test \\
\hline & $\%(n)$ & $\%(n)$ & \\
\hline Any hospital-based mental health care diagnosis & $2.6(2)$ & $13.2(14)$ & $p<0.05$ \\
\hline F10-F19 Mental and behavioral disorders due to psychoactive substance use & $0.0(0)$ & $2.8(3)$ & n.s. \\
\hline F20-F29 Schizophrenia, schizotypal and delusional disorders & $0.0(0)$ & $0.0(0)$ & - \\
\hline F30-F39 Mood disorders & $1.3(1)$ & $6.6(7)$ & n.s. \\
\hline F40-F48 Neurotic, stress-related and somatoform disorders & $1.3(1)$ & $10.4(11)$ & $p<0.05$ \\
\hline $\begin{array}{l}\text { F50-F59 Behavioral syndromes associated with physiological disturbances and physi- } \\
\text { cal factors }\end{array}$ & $0.0(0)$ & $2.8(3)$ & n.s. \\
\hline F60-F69 Disorders of adult personality and behavior & $0.0(0)$ & $1.9(2)$ & n.s. \\
\hline
\end{tabular}

respectively, with mood disorders and for neurotic, stress-related and somatoform disorders at follow-up.

Figure 2a presents odds ratios from a binary logistic regression analysis of mood disorders. In the analysis, mutual adjustments were made for somatic symptoms, sex, and depression and anxiety in adolescence. The presence of $\geq 1$ adolescent somatic symptom was a particularly strong predictor of adult hospital-based mental health care due to mood disorders (OR $=8.45,95 \% \mathrm{CI}$ 1.10-65.03), when mutually adjusting for sex, depression and anxiety in adolescence. When adjusting for the full set of confounders (i.e. adding also conflicts between and with parents, physical abuse, economic hardship, parental unemployment and somatic illness), the estimate was somewhat attenuated and turned non-significant $(\mathrm{OR}=7.06,95 \%$ CI 0.90-55.33, $\mathrm{p}=0.063$ ) (analysis not presented). Since the number of individuals with mood disorders was small, especially among those who did not report any somatic symptoms, this finding should however be interpreted with caution.

Figure $2 \mathrm{~b}$ presents odds ratios from a binary logistic regression analysis of neurotic, stress-related and somatoform disorders. Somatic symptoms were not a significant predictor of neurotic, stress-related and somatoform disorders $(\mathrm{OR}=2.26$, 95\% CI 0.74-6.88). Results from analyses including the full set of confounders (not presented) showed a similar pattern $(\mathrm{OR}=2.12,95 \% \mathrm{CI}$ 0.68-6.61).

\section{Discussion}

This study demonstrated that somatic symptoms in adolescence were associated with long-term severe mental health problems insofar as somatic symptoms did predict adult hospital-based mental health care in adulthood. For individuals with adolescent depression, there was a linear association between the number 

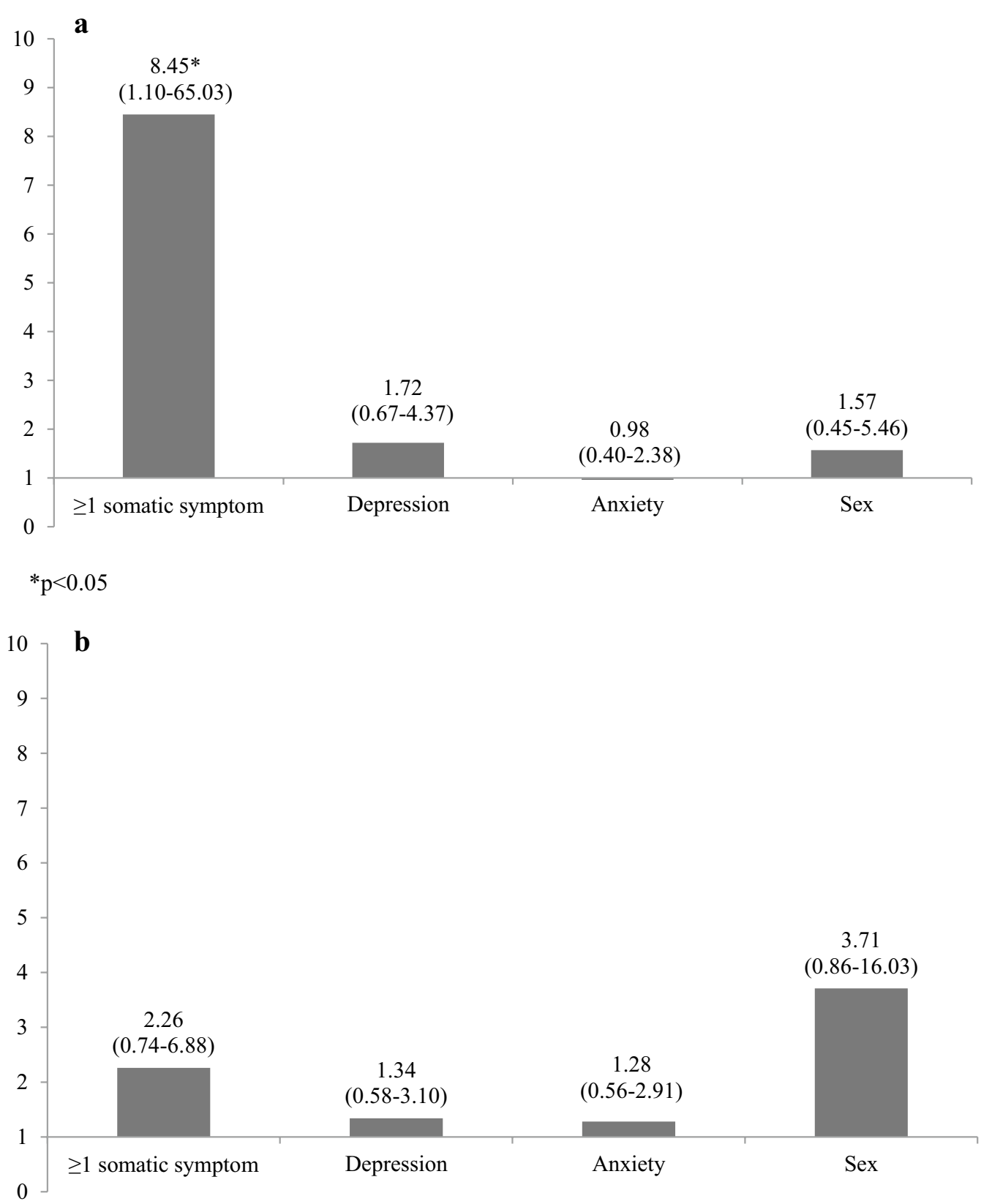

Fig. 2 a Odds ratios with 95\% confidence intervals from a binary logistic regression of hospital-based mental health care for mood disorders in the pooled sample, mutually adjusting for $\geq 1$ somatic symptom, adolescent depression, adolescent anxiety, and sex, $n=375$. b Odds ratios with $95 \%$ confidence intervals from a binary logistic regression of hospital-based mental health care for neurotic, stress-related and somatoform disorders in the pooled sample, mutually adjusting for $\geq 1$ somatic symptom, adolescent depression, adolescent anxiety, and sex, $\mathrm{n}=375$

of somatic symptoms and later use of hospital-based mental health care. For individuals without adolescent depression, any somatic symptom was predictive of later use of hospital-based mental health care.

The findings that somatic symptoms independently predicted later mental health problems reflect those of a previous study using the same baseline data but with follow-up information on depression in adulthood from diagnostic interviews instead of register data on hospital-based mental health care [27]. Thus, the patterns were similar irrespective of whether the mental disorders were captured through interview or through 
diagnoses in clinical settings, implying that the findings are robust. The results are also in line with two recent American studies. Shanahan et al. [25] investigated abdominal pain, muscular pain, and headache with several assessments between 9 and 16 years and anxiety and depression in early adulthood, measured by diagnostic interviews. They found that frequent and recurrent somatic symptoms in childhood predicted anxiety and depression in adulthood after controlling for adolescent anxiety and depression as well as other potential confounders. Shelby et al. [26] found a prediction of functional abdominal pain in childhood and anxiety and depression until young adulthood. By analyzing hospital-based mental health care diagnoses as outcome measures, the current study corroborates the findings of these earlier studies but also extends them by demonstrating that somatic symptoms-in addition to implying risk of developing depression later in lifealso predict a long-term risk of severe mental illness. Furthermore, the results indicate that somatic symptoms might not be less severe than established mental disorders, such as depression and anxiety, in terms of future mental health outcomes and could be an important target for treatment and prevention.

Earlier cross-sectional studies have shown that multiple somatic symptoms are associated with an increased risk of depression as well as depression severity among adolescents in a dose-response relationship [23]. The current study showed that a dose-response relationship also characterizes the long-term risk of hospital-based mental health care, with a particular high risk connected to a high number of somatic symptoms $(\geq 5)$.

Not merely several somatic symptoms but even the presence of few were associated with the outcome in this study. Notably, among the non-depressed adolescents, having one or more somatic symptoms compared to none was associated with a significantly increased risk of later hospital-based mental health care. It should however be noted that while milder symptoms are relatively common even in non-depressed adolescents, in the present study we focused on symptoms with higher severity (as captured through their frequency and intensity).

Furthermore, there might be a stronger link between somatic symptoms and mood disorders than between somatic symptoms and other mental health diagnoses. Having one or more somatic symptoms compared to no somatic symptoms in adolescence predicted hospitalbased care of mood disorders better than adolescent depression and anxiety when mutually adjustments were made. The prediction of hospital-based care for anxiety and somatoform disorders did however not reach statistical significance when adjusting for adolescent depression, anxiety and sex.
The finding that different somatic symptoms were an independent predictor of future hospital-based care of mood disorders has, to our knowledge, not been previously reported, although a Finnish population-based study found that abdominal pain in childhood predicted severe suicidal behavior (suicide and hospital care for suicidal attempts) among men [36].

The mechanisms that link somatic symptoms with future use of hospital-based mental health care for depressive and other disorders might involve different processes. Adolescents with somatic symptoms might have an increased help-seeking behavior which could explain their increased use of hospital-based mental health care in adulthood. Yet, the results from a previous study based on the same data material with adult depression diagnoses based on interviews shows the same pattern, namely, that somatic symptoms predict mental disorders independent from depression and other confounders [27]. This finding speaks against the possibility that help-seeking behavior is an important mediator in the association between somatic symptoms and later hospital-based mental health care. Somatic symptoms could also precipitate unhealthy living conditions that ultimately increase the risk of severe mental illness. For instance, individuals suffering from somatic symptoms might more often fail in higher education [37], and higher education is protective against adverse health outcomes [38]. The link between somatic symptoms and later hospital-based mental health care might also involve biological processes. Both somatic symptoms and emotional distress disorders include dysregulation of the HPA axis and serotonergic pathways [39]. Somatic symptoms could also involve the cytokine system, which underlies inflammatory-based pathways to emotional distress disorders [40]. However, whether there is a dose-response relationship between the number of somatic symptoms and biological markers remains to be shown. Furthermore, somatic symptoms (in particular abdominal pain) could hypothetically be indicative of maladaptive function of the gut involving microbiota, which, in turn, may be involved in regulating physiological systems important in emotional distress disorders [41].

Somatic symptoms have often been regarded as mental disorders by exclusion, as was the case in DSM-IV (but not in DSM-5) for somatoform disorders/somatic symptom disorders [42]. Due to an exclusion of other medical conditions, somatic symptoms might have been regarded as being caused by underlying psychological problems and therefore might not have been the focus of treatment. One implication of such earlier theories might have been a low priority of developing and disseminating effective treatment for somatic symptoms, especially when other problems such as depression and anxiety co-occur. Yet, 
the long-term implications of adolescent somatic symptoms presented in this study indicate that the treatment of somatic symptoms should have a higher priority in mental health services, particularly because emerging data indicate that treatment can be effective $[43,44]$.

\section{Strengths and limitations}

The data and materials had several strengths. The baseline data were population-based, including 2300 adolescents of the same age, with a high participation rate (93\%) in the depression screening. Another advantage was the long follow-up period from adolescence to adulthood. The prospective study design and the use of register data enabled us to follow individuals over time and to avoid the problem of recall bias. The data also provided the opportunity to investigate mental disorders and somatic symptoms at both baseline and follow-up (although neurotic, stress-related and somatoform disorders at followup were grouped together). A limitation was that only about two-thirds of participants in the original investigation were included in the present register-based followup. Yet, the participation rate can be seen as reasonably high in relation to the follow-up period. Furthermore, the attrition rates at follow-up were similar between the depressed and control groups. We assessed bivariate associations between somatic symptoms and later hospital-based mental health care in the two groups separately. To investigate the prediction of somatic symptoms whilst also including a set of potential confounders, we also performed analyses of the pooled sample. This design has limitations since the groups of depressed adolescents and their non-depressed matched peers were different in several respects, as shown in Table 1 . Since only a fraction of non-depressed adolescents were included in the data, the pooled sample is not representative of the original population of 16-17-year-olds in the city of Uppsala. Yet, when assessing the relationship between adolescent somatic symptoms and later hospital-based mental health care, it is of high relevance to control not only for adolescent depression but also for anxiety and other confounders and in this study, this required a pooled sample.

In the present study, we chose to focus on severe mental illness and not on total consumption of mental health care. We did not use information about psychological and pharmacological treatment of mental disorders in general practitioner care, despite the fact that most patients with mental health conditions in Sweden are treated by a general practitioner [45]. Such information could have been of value. A limitation with the strategy of focusing on hospital-based mental health care is also that the actual number of participants who receive such specialized care is relatively small. Another limitation is that a major proportion of adults suffering from mental disorders does not seek or receive adequate treatment. Help-seeking behavior is lower among men than among women, and untreated mental disorders are not uncommon [46]. Hence, it is likely that there are individuals captured in our data who suffer from severe mental disorders without having received hospital-based treatment. This might result in an underestimation of the actual need of adult hospital-based care. Furthermore, the data on hospitalbased outpatient care did not include all registered cases, which implies an underestimation of the total use of hospital-based care and a higher weight of in-patient care compared to out-patient care. Still it seems unlikely that the general findings in relation to our research questions would be affected.

Finally, we lack data on specific somatic diagnoses in adolescence. Hence, we were not able to disentangle whether the association between somatic symptoms in adolescence and hospital-based mental health care in adulthood was due to somatic symptoms with or without a medical explanation. While we did include a measure of hospitalization due to somatic illness or injury in adolescence, this variable might have captured only a portion of the adolescents with somatic illness. Another limitation with this measure is that it was based on adolescents' self-reports.

\section{Conclusions}

Somatic symptoms in adolescence predicted severe mental illness in adulthood as measured by hospital-based care. The prediction remained significant even when adjusted for sex, adolescent depression and anxiety, and other confounders. The presence of at least one somatic symptom compared to none in adolescence was shown to be the strongest predictor of future inpatient care due to mood disorders, surpassing sex, adolescent depression, and anxiety. The findings indicate that adolescents with somatic symptoms need early treatment and extended follow-up due to the increased risk of subsequent poor mental health outcomes.

\section{Additional file}

Additional file 1: Appendix S1. Frequencies of specific somatic symptoms and differences between individuals without and with adolescent depression. Appendix S2. Frequencies of any hospital-based mental health care diagnosis by specific somatic symptoms.

\section{Abbreviations}

DICA-R-A: Diagnostic Interview for Children and Adolescents-RevisedAdolescent; DSM-III-R: Diagnostic and Statistical Manual of Mental DisordersIII-Revised; DSM-IV: Diagnostic and Statistical Manual of Mental Disorders-IV; DSM-5: Diagnostic and Statistical Manual of Mental Disorders-5; HPA: hypothalamic-pituitary-adrenal; ICD-10: International Classification of Diseases-10; MDD: major depressive disorder; SCl: Somatic Symptom Checklist Instrument. 


\section{Authors' contributions}

$\mathrm{HB}$ was responsible for the study concept and the design and drafted the manuscript. HB and SBL performed the statistical analyses. All authors contributed substantially to the interpretation of the data, revised the manuscript critically, and agree to be accountable for all aspects of the work. All authors read and approved the final manuscript.

\section{Author details}

${ }^{1}$ Department of Neuroscience, Child and Adolescent Psychiatry, Uppsala University, Box 593, 75124 Uppsala, Sweden. ${ }^{2}$ Department of Women's and Children's Health, Akademiska University Hospital, 75185 Uppsala, Sweden. ${ }^{3}$ Department of Clinical Science and Education, Södersjukhuset/ Karolinska Institutet, 11883 Stockholm, Sweden. ${ }^{4}$ Department of Public Health Sciences, Stockholm University, 10691 Stockholm, Sweden. ${ }^{5}$ Centre for Psychiatry Research, Department of Clinical Neuroscience, Karolinska Institutet, 17177 Stockholm, Sweden. ${ }^{6}$ Department of Women's \& Children's Health, Center for Neurodevelopmental Disorders at Karolinska Institutet (KIND), Karolinska Institutet, CAP Research Center, Gävlegatan 22B, Floor 8, 113 30 Stockholm, Sweden.

\section{Acknowledgements}

We are grateful to Professor Lars von Knorring for his support and work with the register data, to Professor Anne-Liis von Knorring and Dr. Gunilla Olsson for their work with the baseline study, and to statistician Hans Arinell for advice.

\section{Competing interests}

The authors declare that they have no competing interests.

\section{Availability of data and materials}

Please contact the project manager Dr. Ulf Jonsson for any queries.

\section{Ethics approval and consent to participate}

After a complete description of the study, all included participants gave their written informed consent to the extraction of their register data. The study was approved by the local ethical vetting board of Uppsala University, Sweden.

\section{Funding}

This study was funded by the Söderströmska-Königska Foundation, the Swedish Society of Medicine and the Clas Groschinsky Memorial Foundation.

\section{Publisher's Note}

Springer Nature remains neutral with regard to jurisdictional claims in published maps and institutional affiliations.

Received: 16 October 2017 Accepted: 4 July 2018

Published online: 14 August 2018

\section{References}

1. Poulsen OM, Persson R, Kristiansen J, Andersen LL, Villadsen E, Ørbæk P. Distribution of subjective health complaints, and their association with register based sickness absence in the Danish working population. Scand J Public Health. 2013:41:150-7.

2. Petrie KJ, Faasse K, Crichton F, Grey A. How common are symptoms? Evidence from a New Zealand national telephone survey. BMJ Open. 2014:4:e005374

3. Creed F, Barsky A, Leiknes KA. Epidemiology: prevalence, causes and consequences. In: Creed F, Henningsen P, Fink P, editors. Medically unexplained symptoms, somatisation and bodily distress: developing better clinical services. Cambridge: Cambridge University Press; 2011. p. 1-42.

4. Græsholt-Knudsen T, Skovgaard AM, Jensen JS, Rask CU. Impact of functional somatic symptoms on 5-7-year-olds' healtcare use and costs. Arch Dis Child. 2017. https://doi.org/10.1136/archdischild-2016-311808.

5. Östberg V, Alfvén G, Hjern A. Living conditions and psychosomatic complaints in Swedish schoolchildren. Acta Paediatr. 2006;95:929-34.

6. Steinhausen $\mathrm{HC}$, Winkler Metzke C. Continuity of functional-somatic symptoms from late childhood to young adulthood in a community sample. J Child Psychol Psychiatry. 2007;48:508-13.
7. Swain MS, Henschke N, Kamper SJ, Gobina I, Ottová-Jordan V, Maher CG. An international survey of pain in adolescents. BMC Public Health. 2014;14:447.

8. Modin B, Karvonen S, Rahkonen O, Östberg V. School performance, school segregation, and stress-related symptoms: comparing Helsink and Stockholm. Sch Eff Sch Improv. 2015;26:467-86.

9. Campo JV, Fritsch SL. Somatization in children and adolescents. J Am Acad Child Adolesc Psychiatry. 1994;33:1223-35.

10. Bernstein GA, Massie ED, Thuras PD, Perwien AR, Borchardt CM, Crosby RD. Somatic symptoms in anxious-depressed school refusers. J Am Acad Child Adolesc Psychiatry. 1997;36:661-8.

11. Låftman SB, Östberg V. The pros and cons of social relations. An analysis of adolescents' health complaints. Soc Sci Med. 2006;63:611-23.

12. Larsson BS. Somatic complaints and their relationship to depressive symptoms in Swedish adolescents. J Child Psychol Psychiatry. 1991;32:821-32.

13. Janssens KAM, Rosmalen JGM, Ormel J, van Oort FVA, Oldehinkel AJ. Anxiety and depression are risk factors rather than consequences of functional somatic symptoms in a general population of adolescents: the TRAILS study. J Child Psychol Psychiatry. 2010:51(304):312.

14. Rask CU, Olsen EM, Elberling H, Christensen MF, Ørnbøl E, Fink P, Thomsen $\mathrm{PH}$, Skovgaard AM. Functional somatic symptoms and associated impairment in 5-7-year-old children: the Copenhagen Child Cohort 2000. Eur J Epidemiol. 2009;24:625-34.

15. Dhossche D, Ferdinand R, van der Ende J, Verhulst F. Outcome of selfreported functional-somatic symptoms in a community sample of adolescents. Ann Clin Psychiatry. 2001:13:191-9.

16. Härmä A-M, Kaltiala-Heino R, Rimpelä M, Rantanen P. Are adolescents with frequent pain symptoms more depressed? Scand J Prim Health Care. 2002;20:92-6.

17. Dorn LD, Campo JC, Thato S, Dahl RE, Lewin D, Chandra R, Di Lorenzo C. Psychological comorbidity and stress reactivity in children and adolescents with recurrent abdominal pain and anxiety disorders. J Am Acad Child Adolesc Psychiatry. 2003;42:66-75.

18. Henningsen P, Zimmermann T, Sattel H. Medically unexplained physical symtoms, anxiety, and depression: a meta-analytic review. Psychosom Med. 2003:65:528-33.

19. Haavisto A, Sourander A, Multimäki P, Parkkola K, Santalahti P, Helenius H, Nikalakaros G, Kumpulainen K, Moilanen I, Piha J, Aronen E, Puura K, Linna S-K, Almqvist F. Factors associated with depressive symptoms among 18-year-old boys: a prospective 10-year follow-up study. J Affect Disord. 2004:83:143-54.

20. Santalahti P, Aromaa M, Sourander A, Helenius H, Piha J. Have there been changes in children's psychosomatic symptoms? A 10-year comparison from Finland. Pediatrics. 2005;115:e434-42.

21. Larsson B, Sund AM. Emotional/behavioural, social correlates and oneyear predictors of frequent pains among early adolescents: influences of pain characteristics. Eur J Pain. 2007;11:57-65.

22. Little C, Williams SE, Puzanovova M, Rudzinski ER, Walker LS. Multiple somatic symptoms linked to positive screen for depression in pediatric patients with chronic abdominal pain. J Pediatr Gastroenterol. 2007;44:58-62.

23. Bohman $H$, Jonsson $U$, von Knorring $A-L$, von Knorring $L$, Päären $A$, Olsson G. Somatic symptoms as a marker for severity in adolescent depression. Acta Paediatr. 2010:99:1724-30.

24. Campo JV. Annual research review: functional somatic symptoms and associated anxiety and depression-developmental psychopathology in pediatric practice. J Child Psychol Psychiatry. 2012;53:575-92.

25. Shanahan L, Zucker N, Copeland WE, Bondy CL, Egger HL, Costello EJ. Childhood somatic complaints predict generalized anxiety and depressive disorders during young adulthood in a community sample. Psychol Med. 2015:45:1721-30.

26. Shelby GD, Shirkey KC, Sherman AL, Beck JE, Haman K, Shears AR, Horst SN, Smith CA, Garber J, Walker LS. Functional abdominal pain in childhood and long-term vulnerability to anxiety disorders. Pediatrics. 2013;132:475-82.

27. Bohman H, Jonsson U, Päären A, von Knorring L, Olsson G, von Knorring A-L. Prognostic significance of functional somatic symptoms in adolescence: a 15-year community-based follow-up study of adolescents with depression compared with healthy peers. BMC Psychiatry. 2012;12:90. 
28. Olsson Gl, von Knorring A-L. Adolescent depression: prevalence in Swedish high-school students. Acta Psychiatr Scand. 1999;99:324-31.

29. Beck AT, Ward CH, Mendelson M, Mock J, Erbaugh J. An inventory for measuring depression. Arch Gen Psychiatry. 1961;4:561-71.

30. Reich W, Herjanic B, Welner Z, Gandhy PR. Development of a structured psychiatric interview for children: agreement on diagnosis comparing child and adolescent interviews. J Abnorm Child Psychol. 1982;10:325-36.

31. Jonsson $U$, Bohman $H$, von Knorring $L$, Olsson G, Päären A, von Knorring A-L. Mental health outcome of long-term and episodic adolescent depression: 15-year follow-up of a community sample. J Affect Disord. 2011;130:395-404.

32. Attanasio V, Andrasik F, Blanchard EB, Arena JG. Psychometric properties of the SUNYA revision of the psychosomatic symptom checklist. J Behav Med. 1984;7:247-57.

33. Coddington RD. The significance of life events as etiologic factors in disease of children. I. A survey of professional workers. J Psychosom Res. 1972;16:7-18.

34. Olsson G. Adolescent depression: epidemiology, nosology, life stress, and social network. Doctoral dissertation. Acta Universitatis Upsaliensis. Uppsala: Uppsala University. 1998.

35. Gilman SE, Kawachi I, Fitzmaurice GM, Buka SL. Socioeconomic status in childhood and the lifetime risk of major depression. Int J Epidemiol. 2002;31:359-67

36. Luntamo T, Sourander A, Gyllenberg D, Sillanmäki L, Aromaa M, Tamminen T, Kumpulainen K, Moilanen I, Piha J. Do headache and abdominal pain in childhood predict suicides and severe suicide attempts? Finnish nationwide 1981 birth cohort study. Child Psychiatry Hum Dev. 2014:45:110-8.

37. Låftman SB, Magnusson C. Do health complaints in adolescence negatively predict the chance of entering tertiary education in young adulthood? Scand J Public Health. 2017. https://doi.org/10.1177/14034 94817713649.
38. The Marmot M, Syndrome Status. How social standing affects our health and longevity. New York: Henry Holt; 2004.

39. Rief W, Hennings A, Riemer S, Euteneuer F. Psychobiological differences between depression and somatization. J Psychosom Res. 2010;68:495-502.

40. Maes M, Twisk FN, Ringel K. Inflammatory and cell-mediated immune biomarkers in myalgic encephalomyelitis/chronic fatigue syndrome and depression: inflammatory markers are higher in myalgic encephalomyelitis/chronic fatigue syndrome than in depression. Psychother Psychosom. 2012;81:286-95.

41. Dinan TG, Cryan JF. Regulation of the stress response by the gut microbiota: implications for Psychoneuroendocrinology. Psychoneuroendocrinology. 2012;37:1369-78.

42. Mayou R, Kirmayer L, Simon G, Kroenke K, Sharpe M. Somatoform disorders: time for a new approach in DSM-V. Am J Psychiatry. 2005;162:847-55.

43. Duberg A, Hagberg L, Sunvisson H, Möller M. Influencing self-rated health among adolescent girls with dance intervention: a randomized controlled trial. JAMA Pediatr. 2013;167:27-31.

44. Bonnert M, Olén $\mathrm{O}$, Lalouni M, Benninga MA, Bottai M, Engelbrektsson J, Hedman E, Lenhard F, Melin B, Simrén M, Vigerland S, Serlachius E, Ljótsson B. Internet-delivered cognitive behaviour therapy for adolescents with irritable bowel syndrome: a randomized controlled trial. Am J Gastroenterol. 2017;112:152-62.

45. The National Board of Health and Welfare. Praxis vid vård av patienter med depression och ångest [Practice in care of patients with depression and anxiety]. Stockholm: The National Board of Health and Welfare; 2009.

46. Rutz W, Wallinder J, von Knorring L, Rihmer Z, Pihlgren H. Prevention of depression and suicide by education and medication: impact on male suicidality. An update from the Gotland study. Int J Psychiatry Clin Pract. 1997;1:39-46.
Ready to submit your research? Choose BMC and benefit from:

- fast, convenient online submission

- thorough peer review by experienced researchers in your field

- rapid publication on acceptance

- support for research data, including large and complex data types

- gold Open Access which fosters wider collaboration and increased citations

- maximum visibility for your research: over $100 \mathrm{M}$ website views per year

At $\mathrm{BMC}$, research is always in progress.

Learn more biomedcentral.com/submissions 